\title{
Application potential of modulation of cyclooxygenase-2 activity: a cognitive approach
}

Katarzyna Stachowicz

Department of Neurobiology, Maj Institute of Pharmacology, Polish Academy of Sciences, Kraków, Poland

Abstract

Cognitive functions of the brain depend largely on the condition of the cell membranes and the proportion of fatty acids. It is known and accepted that arachidonic acid (AA) is one of the main $\omega-6$ fatty acids (phospholipids) in brain cells. Metabolism of that fatty acid depends on the functionality and presence of cyclooxygenase (COX). COX is a primary enzyme in the cycle of transformation of AA to prostanoids, which may mediate response of immune cells, contributing to brain function and cognition. Two COX isoforms (COX-1 and COX-2), as well as a splice variant (COX-3), have been detected in the brain. Findings released in the last decade showed that COX-2 may play an important role in cognition. There are many preclinical and clinical reports showing its engagement in Alzheimer disease, spatial learning, and plasticity. This manuscript focuses on summarizing the above-mentioned discoveries.

Keywords

Received: 18.12.2020, Accepted: 07.06.2021

Nervous tissue is characterized by high lipid content. Omega-6 $(\omega-6)$ and $\omega-3$, polyunsaturated fatty acids (PUFAs), represent the primary PUFAs in the brain. They are delivered to the body through food. Omega-6 PUFAs can be found in, among other foods, soybeans, corn, sunflower oils, nuts, meat, and eggs, while $\omega-3$ PUFAs are present in fish (salmon, mackerel, sardines) as well as in meat, eggs, flaxseed oils, soybeans, and yogurts [1, 2]. Arachidonic acid (AA) is the main PUFA in the $\omega-6$ metabolic pathway. AA can be delivered to the body in two ways: with food or as a derivative of linoleic acid (LA). Foods containing AA include eggs, salmon, and tuna. Besides, LA can be provided by the consumption of vegetable oils such as corn, soybean, and sunflower (safflower) oil, and is also present in walnuts [3]. In addition to the exogenous source of $A A$, it can be obtained from an endogenous source, which is 2-arachidonoylglycerol
(2-AG), the endogenous CB1 receptor agonist [3, 4]. AA is a 20-carbon PUFA with four double bonds (20:4). Omega-3 uses a separate metabolic pathway with the following main PUFAs being generated: alpha-linolenic acid (ALA), eicosapentaenoic acid (EPA), and the end product of that pathway, that is, docosahexaenoic acid (DHA) [1]. The right balance between these two PUFA pathways is responsible for the proper functioning of the brain [1]. A schematic diagram of omega-6 and omega-3 PUFA pathways are shown in Fig. 1.

Studies conducted in recent years have shown that diet has a significant effect on PUFA composition in the brain. It was documented that imbalanced PUFA intake, with an increased level of $\omega-6$ to the disadvantage of $\omega-3$, caused atherosclerosis, impoverishment of cognitive functions, and was correlated with Alzheimer disease [1]. On the other hand, an increase of $\omega-3$ PUFAs in the diet was associated 
Omega-6 (w-6)

Diet: soybeans, corn, sunflower oils, nuts, meat, eggs

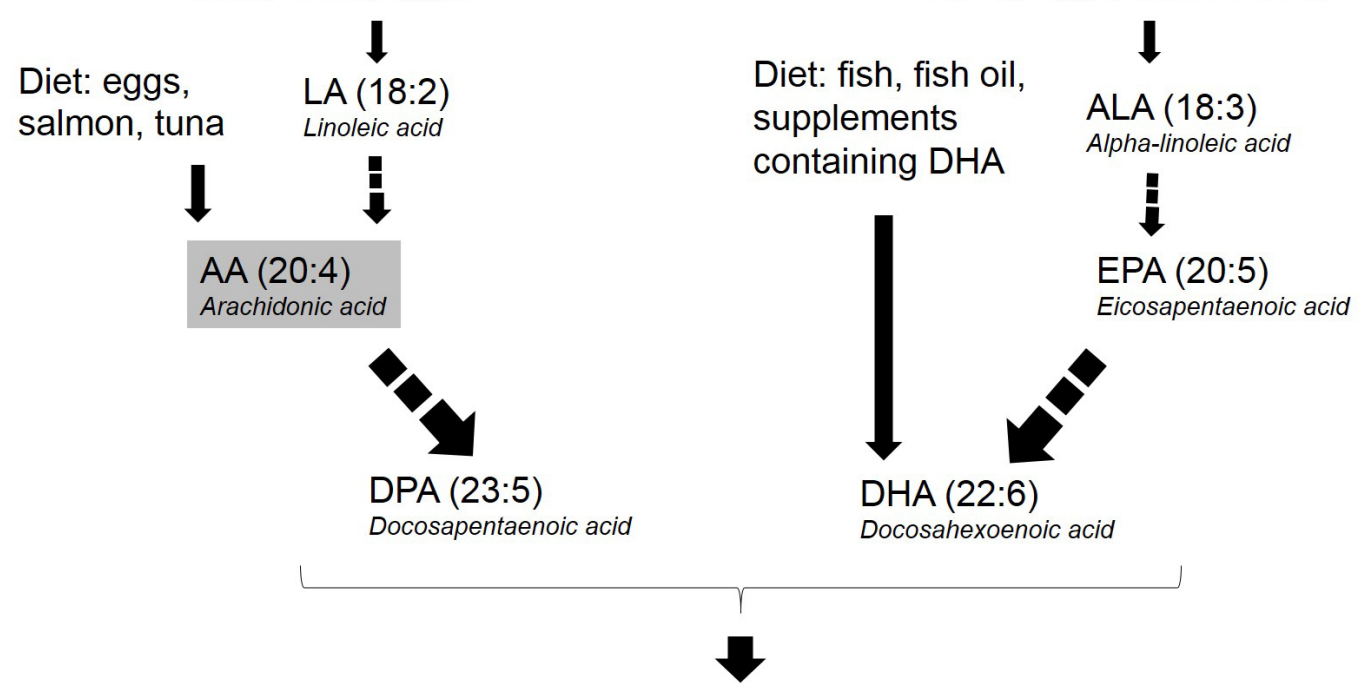

Omega-3 (w-3)

Diet: salmon, mackerel, anchovies, sardines meat, eggs, flaxseed oil, soybeans, yogurts

Diet: fish, fish oil, supplements $\operatorname{ALA}(18: 3)$

pha-linoleic acid

EPA (20:5)

Eicosapentaenoic acid

$\omega-6: \omega-3$ balance

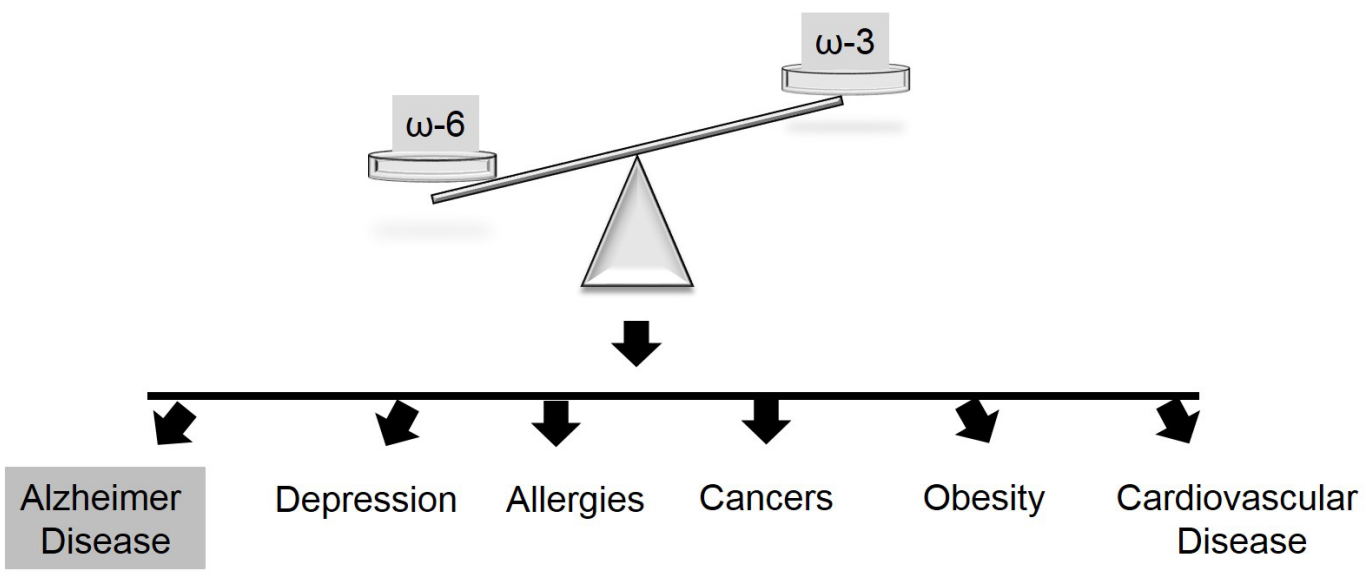

Figure 1. Schematic diagram of omega- 6 and omega-3 PUFA pathways, taking into account diet and diseases in case of imbalance between omega- 6 and omega-3.

with improvement of cognitive functions [1]. Importantly, it was documented that diet favoring $\omega-6$ PUFAs, besides hampering cognitive functions, is connected with an increase of pro-inflammatory mediators (e.g., eicosanoids and interleukins, or IL) [1]. Taking into account the facts presented before, it is very important to understand the mechanisms involved in the transformation of the primary $\omega-6$ PUFA, that is, AA. AA released from the cell membrane under the influence of certain factors, such as stress or inflammation, is subject to the action of COX, which triggers the production of eicosanoids, the main mediators of inflammation in the body. Therefore, it is extremely important both to learn more about the aforementioned mechanisms, as it has been proven that they significantly affect mental health and cognitive abilities, and to elucidate the role of COX - a key enzyme involved in that pathway.

\section{Characterization of COX enzymes}

Cyclooxygenases (COX) - also called prostaglandin $\mathrm{H}$ synthases (PGHS) - exist as two isoforms: COX-1 and COX2. Additionally, a splice variant has been identified, which is COX-3 [5, 6, 7]. Both COX isoforms (COX-1 and COX-2) have 
a very similar primary protein structure. Cyclooxygenase homodimers - with subunit molecular masses of about 70 $72 \mathrm{kDa}$ - consist of the following domains: epidermal growth factor domain, membrane-binding domain, and catalytic domain [6, 7]. The last one comprises two active sites: cyclooxygenase and peroxidase sites. These sites represent two physically distinct elements [6, 7]. The peroxidase site is positioned at the outside of the enzyme and contains heme. A heme prosthetic group is non-covalently bound to the protein and can be reversibly detached, which in turn affects COX activity [6,7]. The cyclooxygenase active site is linked to the membrane thanks to hydrophobic channels located inside the catalytic domain. Access to the channel is provided by the following residues: Arg-120, Tyr-355 and Glu-524 [6, 7]. COX-2 additionally has a side pocket located over the above-mentioned residues - with Val-523 and Arg-513, which makes its active site nearly $20 \%$ larger as compared with COX-1 [6, 7]. Cyclooxygenase secondary and tertiary structure depends on the presence of disulfide bridges [5]. COX is activated through hydration achieved by attaching hydrophobic groups [5]. AA and DGLA (dihomo- $\gamma-$ linolenic acid) provide substrates for COX-1. COX-2, besides the above-listed substances, acts on ALA ( $\alpha$-linolenic acid) and EPA (eicosapentoenoic acid). AA binds to COX-1 in Tyr385, Arg-120, and to COX-2 in Tyr-348, Gly-533 [5].

Despite $60 \%$ sequence similarity, COX-1 and COX-2 fulfill different functions and display distinct localization [6, 7]. In humans, the gene encoding COX-1 is located on chromosome 9 , while the one responsible for COX-2 expression on chromosome 1 [5]. COX-1 is subject to constitutive expression in a wide range of tissues, where it plays a major role in maintaining homeostasis. The gene encoding COX-1 belongs to housekeeping genes and is devoid of TATA box in the 5' region controlling transcription initiation [5]. COX2 isoform is produced as a response to pathophysiological factors, such as cytokines, inflammation, or mental stress $[6,7]$. COX-2 is constitutively expressed in the kidney and brain - predominantly in the hippocampus, hypothalamus, cortex, and spinal cord [6, 7]. In brain tissue it is expressed in neuronal cell bodies, dendrites and astrocytes [5, 8], where it performs a substantial role in synaptic plasticity [8, $9,10]$. Induced COX-2 expression as a response to synaptic stimulation by glutamate may increase in neurons [11]. In response to neuroinflammation, COX-2 expression may increase in astroglia and microglia [11]. Both COX isoforms were found on the inner side of the endoplasmic reticulum,

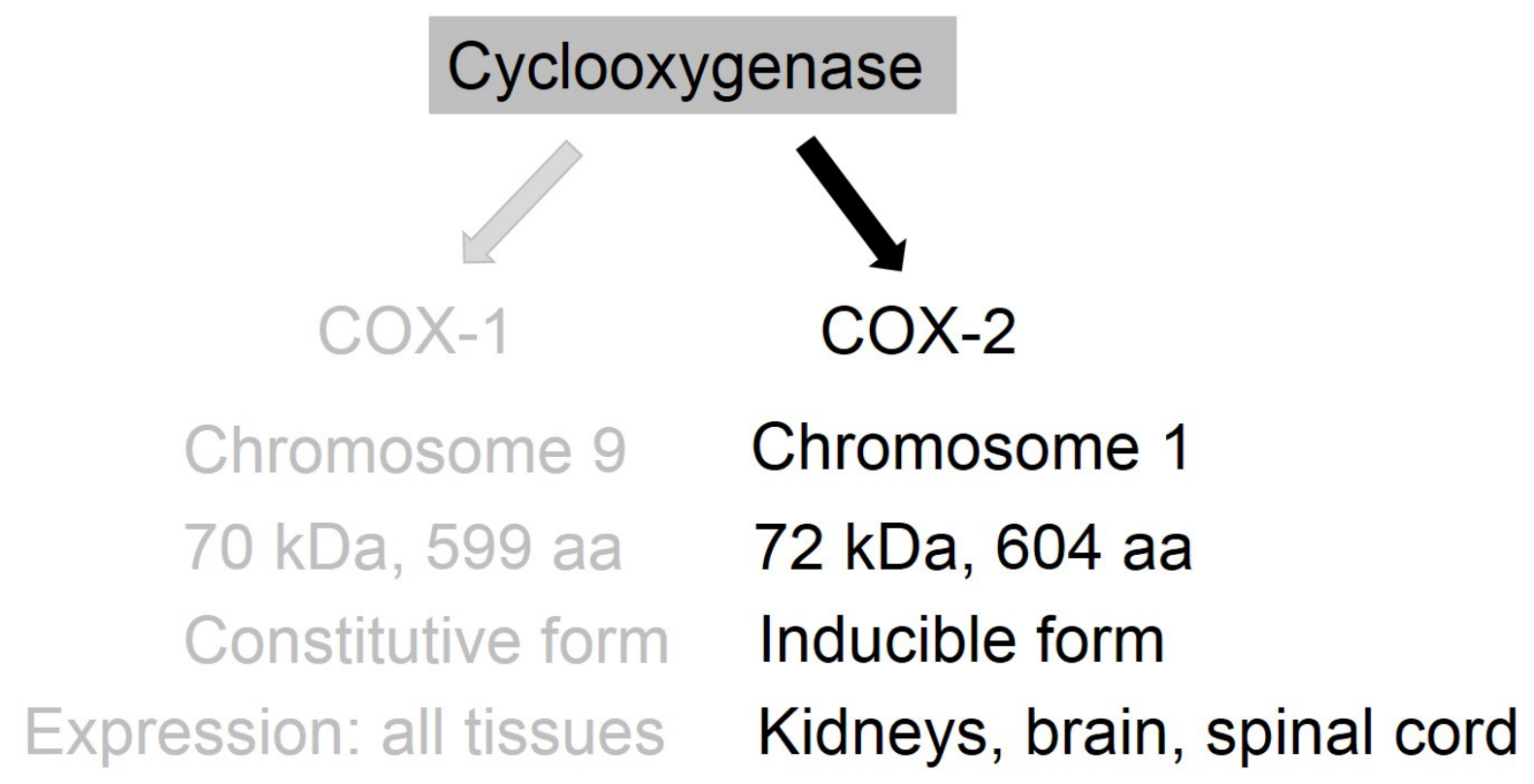

\section{Inner surface of the endoplasmic reticulum both sides of the nuclear sheath}

Figure 2. Basic differences and similarities between COX isoenzymes (COX-1 and COX-2). Cellular and organ expression. 
as well as outside and inside the nuclear sheath [5]. For comparison of COX isoforms see Fig. 2.

COX enzymes are responsible for forming prostanoids (prostaglandin PG, prostacyclin PGI and thromboxane TX) from AA [6, 7]. AA may also undergo transformation to leukotrienes in the lipoxygenase pathway, or to epoxides via cytochrome P450 [5]. The release of AA from the phospholipids in the cell membrane requires phospholipase activity. There phospholipases are generating free AA from cell membranes [3]: phospholipase A2 (PLA2), phospholipase $C$ (PLC), and phospholipase D (PLD). PLA2 releases AA by hydrolysis of the sn-2 position of the phospholipid, while PLC and PLD do so during diacylglycerol (DAG) generating transformations [3]. The initial stage of COX action consists in incorporating two $\mathrm{O}_{2}$ molecules to $A A$ and generating PGG2 [6], which involves a cyclooxygenase active site (located in the catalytic domain). PGG2 - unstable 20 carbon hydroperoxyl-endoperoxide - moves to the peroxidase active site. Activation of COX peroxidase is essential during the second stage of prostanoid synthesis, which involves $\mathrm{PGH} 2$ production, mediated by electron transfer, and reduction to alcohol $[6,7]$. That phase is contingent on the presence of an

\section{Phospholipid bilayer}
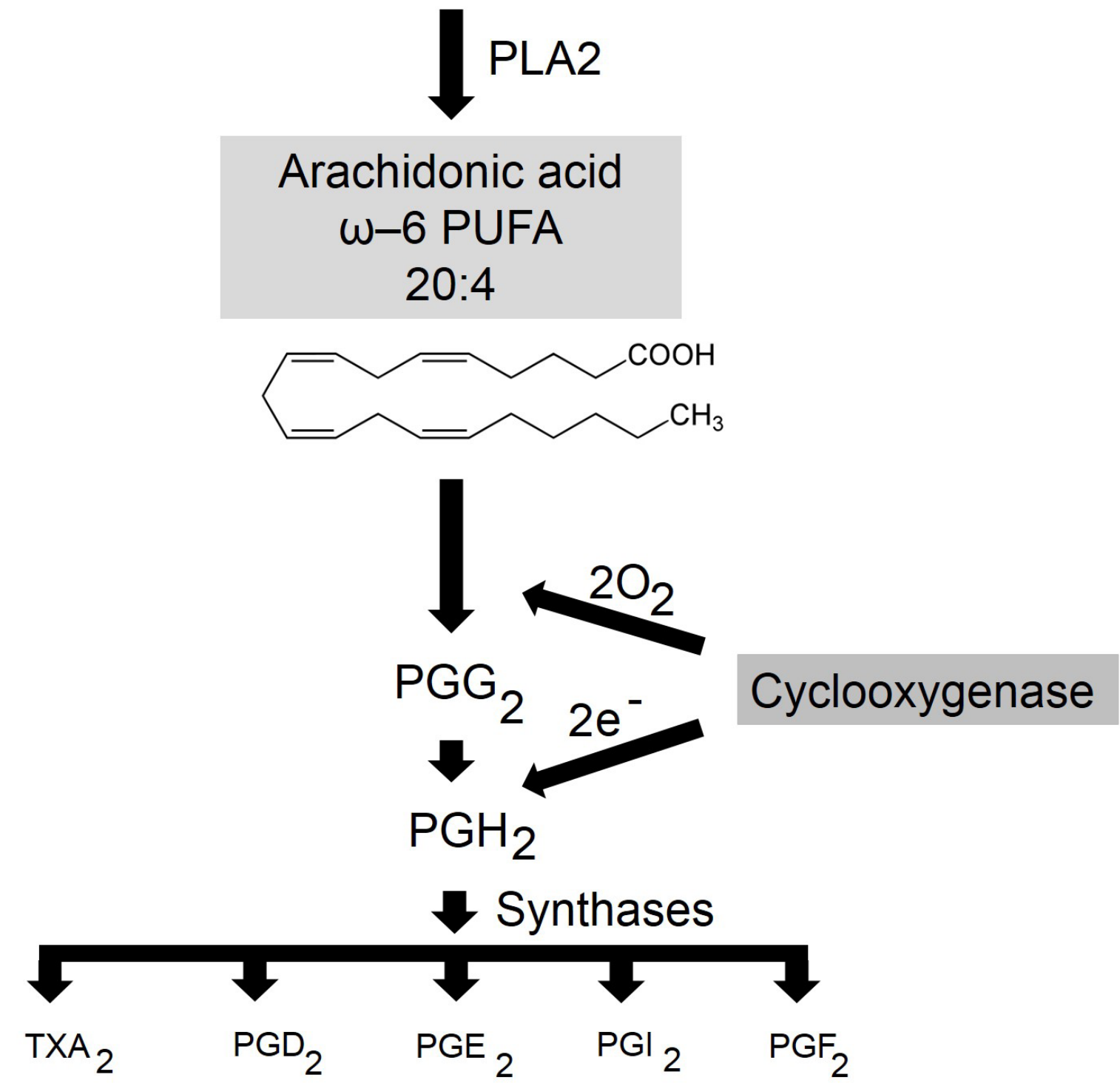

Figure 3. Participation of $C O X$ in prostanoid biosynthesis and transformations. $P G G_{2}$ (prostaglandin $G_{2}$ ) and $P G H_{2}\left(p r o s t a g l a n d i n ~ H_{2}\right)$ as products of COX activity. 
exogenous reductant [7]. Depending on which enzymes and non-enzymatic mechanisms are activated in the subsequent stages of AA transformation, TX, PG, and PGI are formed $[3,11,12]$. Among the prostanoids, this one, belonging to prostaglandin E2 (PGE2), is considered to be the major metabolite of the COX-2 activity. PGE2 activation regulates synaptic plasticity [3, 11, 12], so we will focus on this path in the following sections of this article. A schematic diagram of the PLA2/AA/COX cascade leading to prostanoid generation is presented in Fig. 3.

COX is a heme peroxidase, with heme being bound to the peroxidase active site at the base of the shallow cleft [13]. One iron in the redox center is coordinated to imidazole nitrogen of His-388 [7, 13]. The second one is attached to a water molecule via Tyr-504. In COX His-207 is positioned above the iron particle and is responsible for mediating the removal of a proton from the hydroperoxide substrate and subsequent protonation of the alkoxide ion. The series of complicated reactions leads to producing oxyferryl intermediate and alcohol [7]. To learn more about this process, refer to Kudalkar et al. [7]. Interestingly, mutation of either His-388 or His-207 decreases COX peroxidase activity [7]. It is worth mentioning that cyclooxygenase activity of COX is conditional upon the peroxidase activity site, but it does not work the opposite way [7]. COX activation requires lipid peroxide-dependent oxidation of the heme group. At the subsequent stage, oxidized heme mediates tyrosine oxidation in the cyclooxygenase site. Then, the hydrogen atom is disconnected from $A A$ and the $A A$ radical reacts with $\mathrm{O}_{2}$, which generates PGG2 - a lipid peroxidase. At low AA concentration, the quantity of lipid peroxidase necessary to stimulate cyclooxygenase activity of COX-1 is ten times greater than in the case of COX-2, which indicates that these two isoforms are subject to distinct regulation mechanisms [14].

Substances capable of inhibiting COX enzymatic activity have been classified, among others, into two categories: selective and non-selective compounds. Inhibition of COX enzymatic activity by aspirin (acetylsalicylic acid) is achieved through covalent modification of COX-1, while in the case of reversible COX-2 inhibitors via noncovalent binding [13]. Aspirin - 2-(acetyloxy)benzoic acid - is the only substance applied in the clinic to irreversibly inactivate COX-1 and COX-2 by modifying covalently COX active sites in a timedependent manner [15]. Aspirin acetylates COX at Ser-530 [15]. Despite the fact that acetylation of Ser- 530 by aspirin leads to non-covalent modification of both COX-1 and COX2, this COX inhibitor is 10 to 100 times more potent towards COX-1 [15]. Indomethacin - a non-selective COX inhibitor -blocks COX function, through a progressive and timedependent process [15]. After protracted incubation with the enzyme, indomethacin can be retrieved intact, which implies the involvement of conformational alterations rather than covalent interaction. The inhibitory property of indomethacin depends on ion pairing and/or binding hydrogen to Arg120 [15]. Concerning selective COX-2 inhibitors, such as celecoxib, these substances act through a three-step kinetic mechanism. Selective COX-2 inhibition is associated with the last step of that mechanism, which leads to generation of a tightly bound complex. The dissociation rate constant is used as a selectivity measure [15]. With regard to COX-2 inhibitors, Arg-120 is critical for time-dependent inhibition [15, 16].

COX inhibitors found application as anti-inflammatory, analgesic, and anticoagulant substances. Their potential in mood and cognitive functions has also been documented [17]. The findings published in recent years point towards participation of COX, especially COX-2, in plastic processes in the brain as well as memory formation. Some research indicates that COX-2 can possibly carry out more important functions that the one originally identified. Above all, there are suggestions that COX-2 is not only capable of oxygenating $\omega-6$ PUFAs such as AA, but also those involved in the $\omega-3$ pathway [13]. The research results published by Smith and Langenbach [14] were among the first to challenge the widely accepted notion that COX-2 is only induced in response to inflammatory stimuli, suggesting a much wider involvement of COX-2. This view was supported by research conducted on COX-1 and COX-2 knockout mice [18, 19, 20, 21]. The author discovered that mice devoid of constitutive COX forms led a normal healthy life, while the animals lacking inducible form deficiency suffered more frequent malformations and displayed a higher mortality rate [14]. As far as the latest research is concerned, the role of COX-2 in cognition and brain plasticity has been confirmed both in basic and clinical trials involving selective COX-2 inhibitors. To sum up all available data, it appears that when synaptic plasticity is concerned COX-2 is much more critical than it was initially assumed to be. The most significant discoveries in that matter will be reviewed in the subsequent sections of the article.

\section{The influence of COX-2 on cognitive and plastic changes in the brain}

The involvement of COX-2 in cognition and plastic changes in the brain was documented in many different fields of research. Learning and memorizing, as a basis of cognition, need consolidation. A blockade of COX-2 enzyme is disruptive for the consolidation process. Teather et al. [22] used a whole range of COX inhibitors (indomethacin, a nonselective COX inhibitor; piroxicam, a nonselective COX inhibitor; NS398, a COX-2 selective inhibitor) in an attempt to distinguish between cognitive memory and habit formation in the Morris water test in rats [22]. It turned out that post- 
training injection (15 min.) of indomethacin and NS398, but not piroxicam, impaired hippocampal-dependent memory formation in the hidden platform test [22]. These effects were not observed when injections were done $2 \mathrm{~h}$ after the training. What is more, the effects were not noted in the dorsal-striatal area dependent on the visible platform task [22]. The authors concluded that hippocampal memory formation is connected with high expression of COX-2 in the hippocampus, in contrast to the striatum [22]. Active participation of COX-2 in memory formation has also been shown in the case of long-term memory formation in the Barnes maze [23], a test equivalent to the water Morris but less stressful for animals [24]. It was documented that imipramine, a known antidepressant, has potential to disrupt memory tasks, while co-treatment with NS398 (a selective COX-2 inhibitor) is capable of reversing those changes in mice [23]. More specifically, when mice were injected with imipramine, they were not able to remember training after 10 days of retention. However, cotreatment with a COX-2 inhibitor restored that ability [23, 25]. The studies conducted by Teather et al. [22] and Stachowicz et al. [23] resulted in similar conclusions. The authors point to a special role of cytosolic phospholipase A2 (CPLA2) in the process of memory formation [23]. During the process of memorizing, $\mathrm{Ca}^{2+}$ at the intracellular level increases. That action represents the first step of $\mathrm{N}$-methyl-D-aspartate (NMDA) receptor activation and translocation of CPLA2 to the membrane $[6,23]$. cPLA2 hydrolyses membrane phospholipids and initiates AA production $[6,23]$. It is a first stage of membrane changes that result in $G$ protein-coupled receptor (GPCR) engagement and neurotransmission changes (adrenergic, glutamatergic, and serotoninergic), or activation of the alternative pathway (platelet activating factor, or PAF) [26]. In the case of NMDA receptor activation, bidirectional regulation between NMDA and COX-2 was documented [27, 28]. Furthermore, impairment of memory formation in the Morris water maze was detected after NMDA antagonist AP5 use [29]. AA released from membrane phospholipids is a PG precursor that modulates neurotransmitters [11, 12]. More specifically, the mechanism of synaptic plasticity with COX-2 involvement is as follows: PGE2 induced by COX-2 activity [3, 11, 12] travels retrogradely from the postsynaptic site to the presynaptic part to bind the EP2 receptor [11]. Through an undiscovered mechanism, the activation of Glu exocytosis occurs [11]. This undefined mechanism may be related to the direct effect of COX-2 on mGluR7 in a presynaptic part, a phenomenon we recently discovered [30]. Parallel to this, the release of Glu is limited when 2-AG is converted to a glycerol ester by COX-2, which in turn will limit stimulation of the $\mathrm{CB} 1$ receptor regulating $\mathrm{Ca}^{2+}$ input and exocytosis of Glu at the presynaptic axon terminal [11]. Glu promotes synaptic plasticity through the NMDA postsynaptic receptor and at the same time promotes COX-2 expression [11]. Neuronal deafferentation or inhibition of the NMDA receptor regulates COX-2 expression [10]. Long-term potentiation (LTP), considered a neurobiological learning model, has been shown to be associated with an increase in COX-2 expression [31]. The first report showing that COX-2 expression may be associated with NMDA receptor activity comes from the results of Yamagata et al. [10]. These authors showed that MK801 (NMDA receptor antagonist) blocks the increase in COX-2 expression [10]. LTP in the hippocampus was blocked when the selective COX-2 inhibitor NS398 was used (in vitro); the adding of PGE2 reversed that effect [32]. Furthermore, the use of COX-2 inhibitors (celecoxib, NS398) impaired acquisition and consolidation of the water maze memory via ERK/c-FOS signaling [31]. Both COX-2 inhibition and cPLA2 knockdown blocks LTD [11]. All factors mentioned above are involved in changes in synapse action, transition, timing of Glu release, long-term potentiation (LTP) and synapse remodeling $[6,23,28]$. Genetic knockout of COX-2 produces, among others, autism-related behavior that influences cognitive abilities [33].

Synaptic plasticity can also be understood as a purely physical process of synapse formation, remodeling, and dendritic changes. PGs are known factors affecting actin remodeling in the cytoskeleton, influencing the shape of spines and dendrites, and thus synaptic efficacy and information storage [22]. Connections between synapses and their shapes are guaranteed by cell adhesion molecules - for instance, Down syndrome cell adhesion molecule (DSCAM) $[34,35]$. DSCAM is a representative of the immunoglobulin superfamily of cell adhesion molecules, with documented engagement in cognitive processes [34, 35]. DSCAM takes part in a process of synapse strengthening and timing of synaptic Glu transmission [34]. Our unpublished results indicated that DSCAM level in the mouse brain undergoes COX-2 regulation [manuscript in preparation]. Repeated injections of NS398, a selective COX-2 inhibitor, resulted in changes in DSCAM level in the hippocampus of mice and this effect was time-dependent [manuscript in preparation]. DSCAM - via postsynaptic density proteins/scaffolding proteins of dendrites, such as PSD-95 - may modulate synapse function. That is why this mechanism should be considered in the case of discussion on how COX-2 influences synapse function, which is additionally supported by the fact that both proteins co-localize in dendrites [5, 35]. This is reinforced when considering that the effects of COX-2 inhibitors on scaffolding proteins were documented [36]. The link between DSCAM and senile plaque formation in Down syndrome (DS) with Alzheimer disease (AD) was proposed [37]. In a model of AD with APP transgenic mice (amyloid precursor protein) use, an increased level of DSCAM protein in the cerebral cortex was found [38]. It seems that DSCAM plays the role closely related to the regulation of oxygen 
metabolism in the cell, when its expression is changed under the influence of COX-2 inhibitors [39]. Our unpublished data documented an increase in the DSCAM protein in $\mathrm{HC}$ following COX-2 inhibition under conditions of LPS-induced cell stress [manuscript in preparation]. At the same time, Cillero-Pastor et al. [39] documented an increase in the level of PGE2 and an increased expression of COX-2 in human chondrocytes using inhibitors of the mitochondrial complex III and V. The observations cited above may be important in understanding the mechanisms regulating synaptic plasticity and in finding new effective therapies to improve memory.

\section{The role of COX-2 in Alzheimer disease}

Alzheimer disease (AD) represents a special case of cognitive abnormalities, classified as neurodegenerative disorders. AD dementia is characterized by cognitive memory dysfunctions - orientation in space, calculation, impairment of speech abilities, and more [40]. The root cause of the AD is unknown. However, a characteristic picture of changes in brain tissue can be observed, such as formation of extracellular deposits of $\beta$-amyloid protein (known as senile plaques) and intracellularly localized hyperphosphorylated tau protein, forming neurofibrillary tangles [40]. All alterations listed above are the cause of $\mathrm{Ca}^{2+}$ homeostasis dysregulation, changes in synapses, and degeneration of neurons [40]. So far, no cure for $A D$ has been discovered. Existing therapies slightly improve cognitive performance at the onset of the disease, but are ineffective at the advanced stages, when brain tissue undergoes degeneration. That is why it is so important to search for mechanisms responsible for the brain's cognitive functions, in order to provide patients with more effective treatment, or prevention of the development of the disease.

There is evidence that mechanisms engaging COX are present in $A D$ brains. An increased expression of $C O X$ in human postmortem AD brains was documented [41]. Hoozemans et al. [41], using immunohistochemistry, tried to mark the level of COX-1 and COX-2 in AD brains and compare it with healthy ones. COX-1 expression was detected in microglial cells, while COX-2 was found in neuronal cells [41]. An increased level of COX suggests that both isoenzymes are implicated in the pathomechanism of AD. Moreover, they may be involved in different cellular processes in the pathogenesis of $A D$ [41]. Interestingly, it looks like COX is actively engaged in the pathomechanism of AD, while different COX-2 levels are detected in $A D$ brains depending on the stage of the

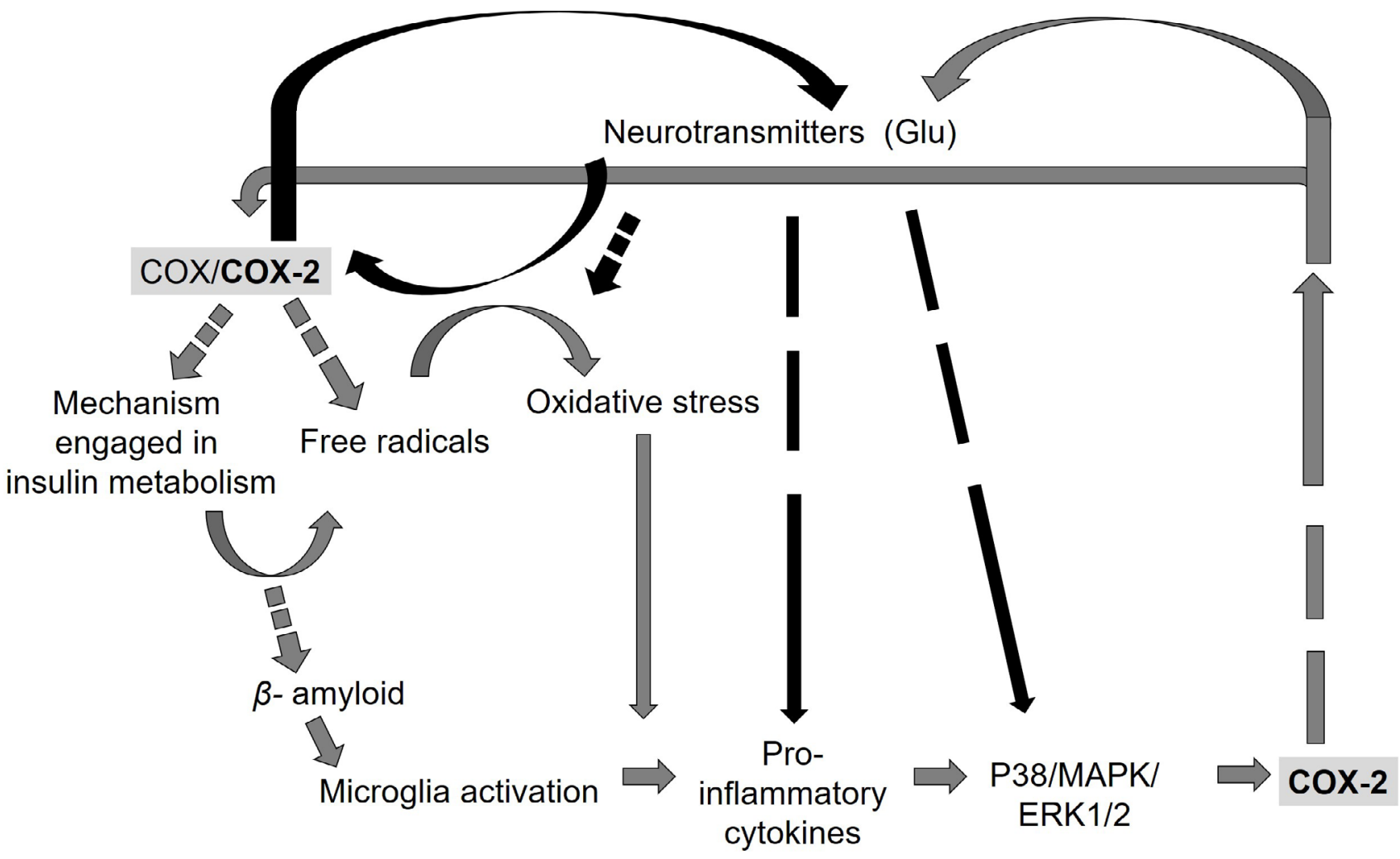

Figure 4. Schematic diagram of pathways influencing COX/COX-2 expression in the pathomechanism of cognitive abnormalities/AD. 
disease. Yemakova et al. [42] detected downregulation of neuronal COX-2 expression in the final stage of $A D$.

An obvious question arises as to how COX could be involved in AD. Elevated COX-2 expression and PGE2 production during pathological conditions is connected with signaling disruption [43]. COX-2 overexpression induces changes in Glu transmission, while a feedback loop is formed where excitotoxic Glu levels drive the mechanisms related to AA production of PG and COX [44]. Glu/COX interaction in the brain has been well established [23, 24, 44]. Moreover, the upregulation of COX-2 followed by $\beta$-amyloid or TNFa upregulation in neurons, astrocytes, or microglia increases by feedback from PG and COX [43]. $\beta$-amyloid induces NF-KB, PKC, ERK p38, MAPK activation. These effects are controlled by COX inhibitors $[43,45]$. In recent years a breakthrough was made in the way we think about the pathomechanism of $A D$. The connection between mechanisms of $A D$ and diabetes mellitus was found [46]. Moreover, it was established that there is a link between mechanisms engaged in cognition and pathways used by COX-2 and insulin-like growth factor 1 (IGF-1). Our results revealed that a COX-2 inhibitor (NS398), active in cognitive studies in rodents, influences IGF-1 level in the periphery [47]. Therefore, we cannot exclude the involvement of the same mechanisms in the center. Further research is necessary to fully understand these mechanisms. Figure 4 is an attempt to summarize known mechanisms involved in cognitive alteration during $A D$ development.

It's worth mentioning that studies conducted by Schaefer et al. [48] drew scientists' attention to the possible participation of DHA in the pathomechanism of $A D$, and especially phosphatidylcholine (PC) DHA [48]. The abovementioned study documented correlation between plasma levels of PC DHA and the risk of developing dementia in the Framingham Heart Study [48].This study showed that higher levels of PC DHA in the plasma of patients participating in the study were associated with a reduced risk of developing dementia in the following years [48]. The above premises indicate that the direction of searching for a therapy improving cognition through the mechanisms that engage COX is appropriate, and that they bring real hope for understanding the pathomechanisms of dysfunctions leading to dementia and/or cognitive problems.

\section{Conclusions and future perspectives}

This manuscript is focused on tracing the signaling paths and mechanisms engaged by COX that may affect cognition and synaptic plasticity. Among the mechanisms discussed, there are those known for a long time, such as AA transformation to PG influencing immunological components. However, quite a new mechanism of COX was found in recent years: the one related to mechanisms of synaptic plasticity outside the immune system. It turns out that COX can affect synaptic plasticity on many levels, from interference with memory formation and consolidation processes, to purely physical conformational changes in synapses. What is more, the role of COX in $A D$ has recently been discussed. The regulation of the level of neurotransmitters via the signal path of PLA2/ AA/COX is of particular interest, as it can translate into the functioning of synaptic connections and ultimately into the perception of the world. Further research is necessary, as it will allow us to understand the importance of signaling pathways related to COX, which may result in the development of effective therapies in the cognitive field.

\section{Abbreviations}

AA - arachidonic acid; AD - Alzheimer disease; ALA - alpha-linolenic acid, a-linolenic acid; APP - amyloid precursor protein; COX - cyclooxygenase; COX-1 cyclooxygenase 1; COX-2 - cyclooxygenase 2; cPLA2 cytosolic phospholipase A2; DGLA - dihomo-y-linolenic acid; DHA - docosahexaenoic acid; DS - Down syndrome; DSCAM - Down syndrome cell adhesion molecule; EPA - eicosapentoenoic acid; FA - fatty acids; Glu - glutamic acid/glutamate; GPCR - G protein-coupled receptor; IL interleukin, LA - linolenic acid; LTP - long-term potentiation; NMDA - N-methyl-D-aspartate; PC - phosphatidylcholine; PG - prostaglandin; PGG2 - prostaglandin G2; PGH2 - prostaglandin H2; PGHS - prostaglandin H synthase; PGI - prostacyclin; PLA2 - phospholipase A2; PUFAs polyunsaturated fatty acids; $\mathbf{T X}$ - thromboxane.

\section{Funding}

This study was supported by the statutory fund of Maj Institute of Pharmacology, PAS, Poland.

\section{Conflict of Interest}

The author has no potential conflicts of interest to declare.

\section{References}

[1] Watson R., Preedy V.: Omega Fatty Acids in Brain and Neurological Health. 2nd Edition. Academic Press, Cambridge MA, 2019.

[2] Zivkovic A.M., Telis N., German J.B., Hammock B.D.: Dietary omega-3 fatty acids aid in the modulation of inflammation and metabolic health. Calif. Agric., 2011; 65: 106-111. 
[3] Hanna V.S., Hafez E.A.: Synopsis of arachidonic acid metabolism: A review. J. Adv. Res., 2018; 11: 23-32.

[4] Lowin T., Straub R.H.: Cannabinoid-based drugs targeting CB1 and TRPV1, the sympathetic nervous system, and arthritis. Arthritis Res. Ther., 2015; 17: 226.

[5] Burdan F., Chałas A., Szumiło J.: Cyclooxygenase and prostanoids: biological implications. Postępy Hig. Med. Dośw., 2006; 60: 129-141.

[6] Fonteh A.N., Wykle R.L.: Arachidonate Remodeling and Inflammation. Birkhäuser Basel, Basel 2004.

[7] Kudalkar S.N., Rouzera C.A., Marnett L.J.: The peroxidase and cyclooxygenase activity of prostaglandin $\mathrm{H}$ synthase. In: Heme Peroxdases, Eds: E. Ravenm, B. Dunford. The Royal Society of Chemistry, London 2016, 245-271.

[8] Strauss K.I.: Antiinflammatory and neuroprotective actions of COX2 inhibitors in the injured brain. Brain Behav. Immun., 2008; 22: 285-298.

[9] Breder C., Dewitt D., Kraig R.P.: Characterization of inducible cyclooxygenase in rat brain. J. Comp. Neurol., 1995; 355: 296-315.

[10] Yamagata K., Andreasson K.I., Kaufmann W.E., Barnes C.A., Worley P.F.: Expression of a mitogen-inducible cyclooxygenase in brain neurons: Regulation by synaptic activity and glucocorticoids. Neuron, 1993; 11: 371-386.

[11] López D.E., Ballaz S.J.: The role of brain cyclooxygenase-2 (Cox2) beyond neuroinflammation: Neuronal homeostasis in memory and anxiety. Mol. Neurobiol., 2020; 57: 5167-5176.

[12] Sang N., Zhang J., Marcheselli V., Bazan N.G., Chen C.: Postsynaptically synthesized prostaglandin E2 (PGE2) modulates hippocampal synaptic transmission via a presynaptic PGE2 EP2 receptor. J. Neurosci., 2005; 25: 9858-9870.

[13] Rouzer C.A., Marnett L.J.: Cyclooxygenases: Structural and functional insights. J. Lipid Res., 2009; 50: S29-S34.

[14] Smith W.L., Langenbach R.: Why there are two cyclooxygenase isozymes. J. Clin. Invest., 2001; 107: 1491-1495.

[15] Blobaum A.L., Marnett L.J.: Structural and functional basis of cyclooxygenase inhibition. J. Med. Chem., 2007; 50: 1425-1441.

[16] Vecchio A.J., Malkowski M.G.: The structure of NS-398 bound to cyclooxygenase-2. J. Struct. Biol., 2011; 176: 254-258.

[17] Müller N.: COX-2 inhibitors, aspirin, and other potential anti-inflammatory treatments for psychiatric disorders. Front. Psychiatry, 2019; $10: 375$.

[18] Dinchuk J.E., Car B.D., Focht R.J., Johnston J.J., Jaffee B.D., Covington M.B., Contel N.R., Eng V.M., Collins R.J., Czerniak P.M., et al.: Renal abnormalities and an altered inflammatory response in mice lacking cyclooxygenase II. Nature, 1995; 378: 406-409.

[19] Langenbach R., Morham S.G., Tiano H.F., Loftin C.D., Ghanayem B.I., Chulada P.C., Mahler J.F., Lee C.A., Goulding E.H., Kluckman K.D., et al.: Prostaglandin synthase 1 gene disruption in mice reduces arachidonic acid-induced inflammation and indomethacininduced gastric ulceration. Cell, 1995; 83: 483-492.
[20] Lim H., Paria B.C., Das S.K., Dinchuk J.E., Langenbach R., Trzaskos J.M., Dey S.K.: Multiple female reproductive failures in cyclooxygenase 2-deficient mice. Cell, 1997; 91: 197-208.

[21] Morham S.G., Langenbach R., Loftin C.D., Tiano H.F., Vouloumanos N., Jennette J.C., Mahler J.F., Kluckman K.D., Ledford A., Lee C.A., Smithies O.: Prostaglandin synthase 2 gene disruption causes severe renal pathology in the mouse. Cell, 1995; 83: 473-482.

[22] Teather L.A., Packard M.G., Bazan N.G.: Post-training cyclooxygenase-2 (COX-2) inhibition impairs memory consolidation. Learn. Mem., 2002; 9: 41-47.

[23] Stachowicz K., Bobula B., Tokarski K.: NS398, a cyclooxygenase-2 inhibitor, reverses memory performance disrupted by imipramine in C57BI/6J mice. Brain Res., 2020; 1734: 146741.

[24] Stachowicz K.: Behavioral consequences of the co-administration of MTEP and the COX-2 inhibitor, NS398 in mice. Part 1. Behav. Brain Res., 2019; 370: 111961.

[25] Harrison F.E., Hosseini A.H., McDonald M.P.: Endogenous anxiety and stress responses in water maze and Barnes maze spatial memory task. Behav. Brain Res., 2009; 198: 247-251.

[26] Jerusalinsky D., Fin C., Quillfeld J.A., Ferreiara M.B., Schmitz P.K., Da Silva R.C., Walz R., Bazan N.G., Medina J.H., Izquierdo I.: Effect of antagonists of platelet-activating factor receptors on memory of inhibitory avoidance in rats. Behav. Neural Biol., 1994; 62: 1-3.

[27] Loza A.M., Elias V., Wong C.P., Ho E., Bermudez M., Magnusson K.R.: Effects of ibuprofen on cognition and NMDA receptor subunit expression across aging. Neuroscience, 2017; 344: 276-292.

[28] Stark D.T., Bazan N.G.: Synaptic and extrasynaptic NMDA receptors differentially modulate neuronal cyclooxygenase-2 function, lipid peroxidation, and neuroprotection. J. Neurosci., 2011; 31: 13710-13721.

[29] Packard M.G., Teather L.A.: Double dissociation of hippocampal and dorsal-striatal memory systems by post-training intracerebral injections of 2-amino-5-phosphonopentanoic acid. Behav. Neurosci., 1997; 111: 545-551.

[30] Stachowicz K., Sowa-Kućma M., Pańczyszyn-Trzewik P., Misztak P., Marciniak M., Bobula B., Tokarski K.: Behavioral consequences of co-administration of MTEP and the COX-2 inhibitor NS398 in mice. Part 2. Neurosci. Lett., 2021; 741: 135435.

[31] Cowley T.R., Fahey B., O'Mara S.M.: COX-2, but not COX-1, activity is necessary for the induction of perforant path long-term potentiation and spatial learning in vivo. Eur. J. Neurosci., 2008; 27: 2999-3008.

[32] Chen C., Magee J.C., Bazan N.G.: Cyclooxygenase-2 regulates prostaglandin E2 signaling in hippocampal long-term synaptic plasticity. J. Neurophysiol., 2002; 87: 2851-2857.

[33] Wong C.T., Bestard-Lorigados I., Crawford D.A.: Autism-related behaviors in the cyclooxygenase-2-deficient mouse model. Genes Brain Behav., 2019; 18: e12506.

[34] Li H.L., Huang B.S., Vishwasrao H., Sutedja N., Chen W., Jin I., Hawkins R.D., Bailey C.H., Kandel E.R.: Dscam mediates trans- 
synaptic interactions for remodeling of glutamate receptors in Aplysia during de novo and learning-related synapse formation. Neuron, 2009; 61: 527-540.

[35] Stachowicz K.: The role of DSCAM in the regulation of synaptic plasticity: Possible involvement in neuropsychiatric disorders. Acta Neurobiol. Exp., 2018; 78: 201-219.

[36] Neuss H., Huang X., Hetfeld B.K., Deva R., Henklein P., Nigam S., Mall J.W., Schwenk W., Dubiel W.: The ubiquitin- and proteasome-dependent degradation of COX-2 is regulated by the COP9 signalosome and differentially influenced by coxibs. J. Mol. Med., 2007; 85: 961-970.

[37] Head E., Lott I.T., Patterson D., Doran E., Haier R.J.: Possible compensatory events in adult Down syndrome brain prior to the development of Alzheimer disease neuropathology: Targets for nonpharmacological intervention. J. Alzheimers Dis., 2007; 11: 61-76.

[38] Jia Y.L., Jing L.J., Li J.Y., Lu J.J., Han R., Wang S.Y., Peng T., Jia Y.J.: Expression and significance of DSCAM in the cerebral cortex of APP transgenic mice. Neurosci. Lett., 2011; 491: 153-157.

[39] Cillero-Pastor B., Caramés B., Lires-Deán M., Vaamonde-García C., Blanco F.J, López-Armada M.J.: Mitochondrial dysfunction activates cyclooxygenase 2 expression in cultured normal human chondrocytes. Arthritis Rheumat., 2008; 58: 2409-2419.

[40] Kubis A.M., Janusz M.: Alzheimer disease: New prospects in therapy and applied experimental models. Postępy Hig. Med. Dośw., 2008; 62: 372-392.

[41] Hoozemans J.J., Rozemuller A.J., Janssen I., De Groot C.J., Veerhuis R., Eikelenboom P.: Cyclooxygenase expression in mi- croglia and neurons in Alzheimer disease and control brain. Acta Neuropathol., 2001; 101: 2-8.

[42] Yermakova A.V., O'Banion M.K.: Downregulation of neuronal cyclooxygenase-2 expression in end stage Alzheimer disease. Neurobiol. Aging, 2001; 22: 823-836.

[43] Medeiros R., Figueiredo C.P., Pandolfo P., Duarte F.S., Prediger R.D., Passos G.F., Calixto J.B.: The role of TNF- $\alpha$ signaling pathway on COX-2 upregulation and cognitive decline induced by $\beta$-amyloid peptide. Behav. Brain Res., 2010; 209: 165-173.

[44] Strauss K.I., Marini A.M.: Cyclooxygenase-2 inhibition protects cultured cerebellar granule neurons from glutamate-mediated cel death. J. Neurotrauma, 2002; 19: 627-638.

[45] Grishin A.V., Wang J., Potoka D.A., Hackam D.J., Upperman J.S., Boyle P., Zamora R., Ford H.R.: Lipopolysaccharide induces cyclooxygenase-2 in intestinal epithelium via a noncanonical p38 MAPK pathway. J. Immunol., 2006; 176: 580-588.

[46] Akter K., Lanza E.A., Martin S.A., Myronyuk N., Rua M., Raffa R.B.: Diabetes mellitus and Alzheimer disease: Shared pathology and treatment? Br. J. Clin. Pharmacol., 2011; 71: 365-376.

[47] Tabecka-Lonczyńska A., Mytych J., Solek P., Kulpa-Greszta M., Jasiewicz P., Sowa-Kućma M., Stachowicz K., Koziorowski M.: IGF-1 as selected growth factor multi-response to antidepressantlike substances activity in C57BL/6J mouse testis model. Acta Histochem., 2021; 123: 151685.

[48] Schaefer E.J., Bongard V., Beiser A.S., Lamon-Fava S., Robins S.J., Au R., Tucker K.L., Kyle D.J., Wilson P.W., Wolf P.A.: Plasma phosphatidylcholine docosahexaenoic acid content and risk of dementia and Alzheimer disease: The Framingham Heart Study. Arch. Neurol., 2006; 63: 1545-1550. 